\title{
Phototherapy in cancer prevention and treatment
}

\begin{abstract}
Phototherapy is a safe, and secure way to destroy cancer cells when light waves of a particular wavelength used with appropriate activating agents. It is usually limited by its nature to illnesses that are skin-deep. Researchers developed a phototherapy method that brings light directly to tumor cells, no matter how deep they are. Phototherapy also has demonstrated promising results in diagnosing and treating various types of cancers. The combination of nanomedical agents and phototherapy modalities has shown better results than individual therapy. Further research should be encouraged to formulate an effective combination of activating agents and phototherapy for the best outcome, to minimize adverse effects and to prevent injury to healthy tissues. This article reviews different types phototherapy methods to treat cancer.
\end{abstract}

Volume 7 Issue I - 2017

\author{
Sebastian Roy,' Brown Tony L ${ }^{2}$ \\ 'Department of Neurology, International Clinical Research \\ Organization, USA \\ 2Columbia College of Physicians and Surgeons, USA
}

Correspondence: Tony L Brown, Department of Neurology, Columbia University, USA, Email tbrown@post.harvard.edu

Received: April 14, 2016 | Published: January 09, 2017

Keywords: photodynamic therapy, LIT, PTT, HSP, inCVAX, OK-432, HMSNs-DOX/ IR825

\section{Introduction}

\section{Phototherapy}

Phototherapy has reached new heights with the technological breakthroughs of the past four decades, such as using lasers as the light sources, and nanoparticles as the sensitizers. Phototherapy has been commonly employed in either diagnosis or therapeutics lately. Phototherapy demonstrates excellent results in targeting the cause of many diseases. It functions by immunologically delivering and chronically releasing diagnostic and therapeutic agents, and by inducing tissue immunological response and enhancing synergizing immunotherapy. Phototherapy can act as an immunological agent by initiating immunological responses resulting in stimulation of innate immune system. It also destructs established tumor cells by selective photochemical and photothermal interactions, release tumor antigens, and create an in situ cancer vaccine. The synergistic effects of Photoimmunotherapy raise the possibility to improve clinical outcomes. We anticipate that shortly there will be significant advances in the area of PIT, with the better understanding of the induction of antitumor immune response by phototherapy and further development of immunotherapy.

\section{Photodynamic therapy}

Photodynamic therapy (PDT) is a minimally invasive therapeutic method for treatment of cancer. It involves three major elements: a targeted low-level visible light, nontoxic photosensitizers (PSs), and oxygen in tumor tissue. The PDT-induced photochemical reaction produces cytotoxic reactive oxygen species (ROS) to exert a selective cytotoxic activity toward malignant cells. ${ }^{1}$ PDT was the first drugdevice combination approved by the US FDA almost two decades ago. Each photosensitizing agent used in PDT has particular absorbance band of the wavelength of the light. In PDT, after administering the photosensitizing agent at the cancer site and the area is irradiated with appropriate light with the corresponding wavelength in the presence of oxygen. Resulting photochemical reaction creates highly reactive singlet oxygen (1O2). This oxygen is highly toxic and leads to cell death via apoptosis or necrosis. ${ }^{1}$ Photofrin ${ }^{\circledR}$, also known as porfimer sodium is an FDA approved photosensitizing agent, used in the management of non-small cell lung cancer and esophageal cancer. ${ }^{2}$ Porfimer sodium has proved effective in relieving symptoms of esophageal cancer when the mass obstructs the esophagus or when it is untreatable with laser alone. Porfimer sodium is also used to treat non-small cell lung cancer in patients when cancer blocks the airway.

\section{Photothermal therapy}

Photothermal therapy (PTT) is a more developed therapeutic method for local treatment of cancer. It uses heat generated from photon energy to destroy tumor cells, which could be highly precise, much less invasive, and rather effective due to the intensive light beam directly focused on the target tumor. ${ }^{3}$ The electromagnetic sources used in heating include near infrared or visible light, radiofrequency waves, microwaves, and ultrasound waves. They induce moderate temperature rise in a particular target tissue to destroy cancer cells. This kind of thermal therapy is efficient in the treatment of patients with solid tumors affecting organs, such as the liver, kidney, lung, adrenal gland, prostate, and bone. ${ }^{4}$ Recently, PTT has drawn greater attention in cancer treatment with the development of nanotechnology-based photothermally sensitizing agents including gold nanoparticles, gold nanorods, carbon nanotubes, graphene and others. Nanomaterials have the high optical absorbance in the NIR region, and they can transfer photo energy of NIR laser and radiofrequency into heat energy at the tumor site to enhance thermal destruction. ${ }^{4}$ The immune response induced by phototherapy. The first direct effect of phototherapy (PDT and PPT) is their cytotoxicity on tumor cells, either induced by reactive singlet oxygen (ROS) production or by temperature increase. The second important effect of PDT or PPT is the increase in immunogenicity of the tumor when it releases damaged associated molecular patterns (DAMPs) and cell death-associated molecular patterns (CDAMPs) that can be detected by the innate immune systems. Phototherapy could enhance Heat Shock Proteins (HSP). HSP expression in the tumor cells, in particular on the cell surface of apoptotic cells, which could be recognized by APCs through toll-like receptor (TLRs), followed by cytokine release via Myd88 and NF$\mathrm{kB}$ signaling pathways. ${ }^{2}$ In particular, phototherapy, as a promising cancer treatment strategy that uses a local, selective photothermal or photochemical interaction on target tumor to release tumor antigens, creates an antigen source that acts as an in situ cancer vaccine. The HSPs stimulates both innate immunity and adaptive immunity and thus enables the immune system to fight the cancer. ${ }^{5}$ 


\section{Photoimmunotherapy}

Phototherapy can induce death of tumor cells and results in the formation of antigens from the process of cell death. This recently formed antigens can stimulate the immune system. Concomitantly, this target treatment strategy has been combined with immunotherapy that can promote antigen uptake and presentation, thus triggering a specific antitumor immunity. Anti-cancer immunotherapies include antibodies, cytokines and other immune-related proteins and peptides. Photoimmunotherapy using the antibody for the delivery of the photosensitizer using a monoclonal antibody (mAb)-targeted PS and red-light are the novel target phototherapy technique. The treatment approach of NIR-PIT not only increased the target efficacy of PS, but also improved the penetration of PDT using NIR light. EGFRs are overexpressed on the cell surface of several cancers including lung, colon, head and neck, and esophageal cancers. When exposed to NIR light, mAbIR700 conjugate could result in rapid and irreversible damage to the cell membrane, leading to necrotic cell death.

\section{Laser immunotherapy}

Laser immunotherapy (LIT) is a combination therapy of phototherapy and immunotherapy, which utilizes a local intervention to induce a systemic antitumor immunity. The two principles underlying laser immunotherapy are (1) induction of the local immune response by the liberated tumor antigens from the local destruction of tumor cells resulting from direct delivery of laser energy into the tumor, (2) the local administration of an immunoadjuvant to elicit a much stronger systemic immune response. The fundamental mechanism behind LIT is the activation of APCs, such as DCs, and subsequent exposure of the activated APCs to tumor antigens in vivo so that a tumor-specific $\mathrm{T}$ cell response is induced. LIT utilizes whole tumor cells as the source of tumor antigens without any ex vivo preparations, and this is considered an in situ autologous cancer vaccine (trademarked as inCVAX). The early phase LIT approach combined three major components: a NIR laser $(805 \mathrm{~nm})$ a light-absorbing agent (ICG) (enhance light absorption in the tumor tissue), and an immunoadjuvant (enhance immune response after the photothermal treatment). A study indicates that local LIT treatment is capable of reducing and eliminating treated primary tumors as well as untreated metastases in the lungs, significantly prolonging patient survival. ${ }^{6}$ New nano-systems could retain both optical properties of nanomaterials and immunological function of adjuvants, representing promising treatment modalities combined with laser irradiation to induce systemic antitumor response through a local intervention.

\section{Polymeric prodrug grafted hollow mesoporous silica nanoparticles}

Polymeric prodrug coated hollow mesoporous silica nanoparticles (HMSNs) with encapsulated near-infrared (NIR) light absorbing dye were used in a study for combined photothermal-chemotherapy. A copolymer integrated with tert-butoxycarbonyl protected hydrazide groups and oligoethylene glycols was initially grafted on the surface of HMSNs via reversible addition-fragmentation chain-transfer (RAFT) polymerization. It was followed by the deprotection to reactivate the hydrazide groups for the conjugation of anticancer drug doxorubicin (DOX). DOX was covalently bound to the polymer substrate by acid-labile hydrazone bond and released quickly in weak acidic environment for chemotherapy. There is a hollow cavity in HMSNs and it was loaded with an NIR absorbing dye IR825 to form the final multifunctional hybrid denoted as HMSNs-DOX/IR825. The hybrid compound exhibited good dispersity stability and high light-to- heat conversion efficiency. Confocal microscopy and flow cytometry analysis showed the hybrid was efficiently taken up by cancer cells, and the conjugated DOX could be released into the cellular environment. Another in vitro cytotoxicity study demonstrated that anticancer activity of HMSNs-DOX/IR825 could be significantly improved by the NIR irradiation, which proved therapeutic efficient through the combination treatment. ${ }^{7}$ Thus, the developed hybrid could be a significant therapeutic agent for the combined photothermalchemotherapy of cancer.

\section{Combination of immunostimulation and phototherapy}

Immunostimulatory agents combined with phototherapy can be divided into two classes: microbial vaccines and cytokines. Microbial agents derived from microbial components, such as OK432 that was derived from penicillin killed streptococcal bacteria and corynebacterium parvum $(\mathrm{CP})$ which is a killed bacterial vaccine, has been used to combine with PDT.The second class of combination therapies concerns the administration of cytokines with PIT . Photofrin-PDT, such as granulocyte colony stimulating factor (G-CSF) on colo 26 and Lewis lung carcinomas tumor models, granulocyte-macrophage colony stimulating factors (GM-CSF) on SCCVII tumor types, and tumor necrosis factor (TNF $\alpha$ ) on SMT-F adenocarcinoma tumor model. ${ }^{7}$ All administration of cytokines can enhance the therapeutic effects. Other phototherapies have developed in combination with cytokines, such as radiofrequency with interleukin 12 (IL-12) in the treatment of murine colon adenocarcinoma, or with IL-17 and IL-15 in the treatment of murine breast tumors.

\section{Conclusion}

Cancer Nanomedicine possesses the versatility required to overcome uniquely some of the most challenging impediments to treatment success when phototherapy alone is used with regular photosensitizers. Also utilizing phototherapy applications with nanoparticles can be the next level of cancer treatment. Research should focus on identifying the appropriate nanoparticles that work well with the phototherapeutic agents for various cancers depending on the stage and location. Phototherapy enhances the effect of nanoparticles

\section{Acknowledgments}

None.

\section{Conflicts of interest}

Authors declare there are no conflicts of interest.

\section{References}

1. Agostinis P, Berg K, Cengel KA, et al.Photodynamic therapy of cancer: An update. CA Cancer J Clin. 2011;61(4):250-281.

2. Guo Y, Zhang Z, Kim DH, et al. Photothermal ablation of pancreatic cancer cells with hybrid iron-oxide core gold-shell nanoparticles. Int $J$ Nanomedicine. 2013;8:3437-3446.

3. Gao S, Zhang M, Zhu X, et al. Apoptotic effects of Photofrin-Diomed 630-PDT on SHEEC human esophageal squamous cancer cells. Int $J$ Clin Exp Med. 2015;8(9):15098-15107.

4. Hamblin MR, Huang YY. Handbook of Photomedicine. CRC Press LLC: USA; 2013.

5. Murshid A, Gong J, Calderwood SK. The Role of Heat Shock Protein in Antigen Cross Presentation. Frontiers in Front Immunol. 2012;3:63. 
6. Chen WR. Laser immunotherapy for cancer treatment. OSA publishing, ATh3L; 2013.

7. Zhang Y, Ang CY, Li M, et al. Polymeric Prodrug Grafted Hollow
Mesoporous Silica Nanoparticles Encapsulating Near-Infrared Absorbing Dye for Potent Combined Photothermal-Chemotherapy. ACS Appl Mater Interfaces. 2016;8(11):6869-6879. 